\title{
Instability of Kinetic Roughening in Sputter-Deposition Growth of Pt on Glass
}

\author{
J. H. Jeffries \\ MSU Graduate Student
}

J.-K. Zuo

Missouri State University

M. M. Craig

Missouri State University

Follow this and additional works at: https://bearworks.missouristate.edu/articles-chhs

\section{Recommended Citation}

Jeffries, J. H., J-K. Zuo, and M. M. Craig. "Instability of kinetic roughening in sputter-deposition growth of Pt on glass." Physical review letters 76, no. 26 (1996): 4931.

This article or document was made available through BearWorks, the institutional repository of Missouri State University. The work contained in it may be protected by copyright and require permission of the copyright holder for reuse or redistribution.

For more information, please contact BearWorks@library.missouristate.edu. 


\title{
Instability of Kinetic Roughening in Sputter-Deposition Growth of Pt on Glass
}

\author{
J.H. Jeffries, ${ }^{1}$ J.-K. Zuo, ${ }^{1}$ and M. M. Craig ${ }^{2}$ \\ ${ }^{1}$ Department of Physics and Astronomy, Southwest Missouri State University, Springfield, Missouri 65804 \\ ${ }^{2}$ Department of Biomedical Sciences, Southwest Missouri State University, Springfield, Missouri 65804
}

(Received 15 January 1996)

\begin{abstract}
Using scanning tunneling microscopy, we have studied the kinetic roughening in the growth of Pt sputter deposited on glass at room temperature for a film thickness range of $15-140 \mathrm{~nm}$. The growth exhibits an irregularly growing mound morphology and shows an instability with anomalous scaling behavior characterized by the $\sqrt{\ln (t)}$ dependence of the local slope, where $t$ is the growth time, and also by the roughness exponent $\alpha \simeq 0.9$ and interface growth exponent $\beta \simeq 0.26$. These characteristics clearly indicate that the growth is consistent with a statistical model of linear diffusion dynamics. [S0031-9007(96)00472-3]
\end{abstract}

PACS numbers: 68.55.Jk, 61.16.Ch, 64.60.Ht, 81.15.Cd

Because of technological importance and fundamental interest, great efforts have been devoted recently to understanding the kinetic roughening of growing surfaces in various growth techniques. There are three main factors determining a growing surface morphology: deposition, desorption, and surface diffusion. A balance among them leads to a hypothetic self-affine scaling behavior of the growing surface in both time and space [1,2]. This means that the film evolution is such that the time and spatial dependent surface profile $h(\mathbf{r}, t)$ is statistically similar to the surface profile $\lambda^{-\alpha} h\left(\lambda \mathbf{r}, \lambda^{\alpha / \beta} t\right)$, where $\lambda$ is a scaling factor, and $\alpha$ and $\beta$ are independent exponents which characterize universality classes of the growth governed by different growth mechanisms [3].

Theoretical treatments of nonequilibrium film growth use phenomenological expansions in the derivatives of $h(\mathbf{r}, t)$ based on symmetry arguments and the continuity principle [3]. For the surface diffusion-driven growth processes where the desorption is negligible, the growth equation is written as [4]

$$
\frac{\partial h}{\partial t}=-\kappa \nabla^{4} h+\lambda \nabla^{2}(\nabla h)^{2}+F+\eta(\mathbf{r}, t),
$$

where $\kappa$ and $\lambda$ are constants, and $\eta$ is a random fluctuation around the average flux $F$, which causes roughening. The scaling exponents $\alpha$ and $\beta$ for this nonlinear equation are solved to be $\alpha=\frac{2}{3}$ and $\beta=\frac{1}{5}$ for the growth on a two-dimensional $(d=2)$ substrate [4]. However, if one neglects the second term $(\lambda=0)$ in Eq. (1), i.e., for the linear equation, the exponents will be $\alpha=1$ and $\beta=\frac{1}{4}$ [5]. The detailed microscopic processes described by the linear and nonlinear equations have been tested by Das Sarma and Ghaisas using Monte Carlo simulations [6]. They found that two kinds of realistic models of $d=2$ correspond to the linear and nonlinear equations, respectively. The one model, corresponding to the linear equation, is that only isolated atoms with the coordination number $N_{c}=1$ can diffuse and will stick at a nearest kink site irreversibly. The other model, corresponding to the nonlinear equation, is that any surface atom with $N_{c} \leq 2$ is able to break its bonds and hops to an adjacent site with increased $N_{c}$. Obviously, the former describes a local diffusion, and the latter describes an intermediate-range diffusion. It is because of the insufficient surface diffusion in the former case that a groove instability is predicted for the growth in which the root-mean-square (rms) local slope increases with time via [7]

$$
\rho(t) \equiv\left\langle(\nabla h)^{2}\right\rangle^{1 / 2}=\sqrt{C \ln (t / \tau)},
$$

where $\tau$ is an initial transition time to the scaling regime and $C$ is a constant. This anomalous scaling behavior has been observed by Yang, Wang, and Lu [8] in a lowtemperature homoepitaxial growth on $\mathrm{Si}(111)$.

Many recent experiments support the existence of dynamic scaling in surface diffusion-driven growth for various systems [9-13]. However, due to experimental uncertainties, the values of the measured exponents $\alpha$ and $\beta$ often range between those predicted for the two models above. Thus, it is not easy to identify whether a growth is governed by the nonlinear or linear equation based just on the measured exponents, even only on one exponent [1416]. The rms local slope is a unique feature that can be used to distinguish the linear diffusion process from the nonlinear one in which the local slope is time invariant in the scaling regime [2].

More recently, it has been argued that self-affine scaling will break down if there is a Schwoebel barrier at the step edge that resists deposited atoms from step-down diffusion [17,18]. In this case, as deposition proceeds, large-scale uniformly sized pyramids with stationary slope will be formed on a singular substrate surface. Such a growth instability has also been observed, but only on single-crystal epitaxial systems [19-22]. Moreover, the effective exponents $\alpha$ and $\beta$ for the Schwoebel barrier model are also predicted to be $\alpha=1$ and $\beta=\frac{1}{4}$ [17]. Thus, our understanding for the kinetic roughening in film growth is still far from complete.

In this Letter we report a scanning tunneling microscopy (STM) study of the growth mechanism and instability for Pt sputter deposition on glass at room temperature. In order to assure asymptotic behavior, film thicknesses in 
the range of 15 to $140 \mathrm{~nm}$ were grown. Since existence of the Schwoebel barrier is unlikely in the polycrystal system with small grains, the measured exponents, $\alpha \sim$ $0.90 \pm 0.02$ and $\beta \sim 0.26 \pm 0.03$, and the observed $\sqrt{\ln (t)}$ dependence of the rms local slope clearly indicate that the growth belongs to the universality class of the linear diffusion dynamics.

Samples were grown in a B370 Microsputter chamber (VCR group) with a base pressure of $2 \times 10^{-6}$ Torr. By a plasma-excited $\mathrm{Ar}^{+}$ion beam, platinum is sputtered off a 99.99\% pure $\mathrm{Pt}$ target onto a clean glass substrate at room temperature with an approximate deposition rate of $6 \AA / \mathrm{min}$. The sample is placed $\sim 3 \mathrm{~cm}$ away from the target and its surface normal is aligned about $45^{\circ}$ with the target surface normal. The sample holder is operated with both spinning and planetary rotation during deposition to eliminate shadowing effects. After growth, the sample is transferred to an ultrahigh vacuum chamber through air for STM analysis. STM imaging utilized electrochemically etched tungsten tips with typical tunneling current $\sim 1 \mathrm{nA}$ and bias voltage $\sim 1 \mathrm{~V}$. Four tungsten tips have been used for each sample to minimize occasional tip geometric artifacts, and the results are consistent within experimental uncertainty. The glass substrate flatness is characterized by an atomic force microscope (AFM), and the measurement yields an rms roughness of $w_{\text {glass }} \sim 0.17 \mathrm{~nm}$, which is approximately 2.5 times less than that of our thinnest film. The film crystallinity is checked by a $\theta-2 \theta$ x-ray diffractometer which, due to the setup, allows only detection of crystallites having crystalline planes parallel to the substrate surface. Two kinds of crystallites [(111) and (100)] are detected and their sizes are 16.5 and $7.5 \mathrm{~nm}$, respectively, for a film of thickness $130 \mathrm{~nm}$. However, only (111) crystallites are detectable with a size of $9.5 \mathrm{~nm}$ for a $60 \mathrm{~nm}$ thick film. In addition, the Pt film is found to wet the glass well in vacuum. This is evidenced by the fact that, for a $3 \mathrm{~nm}$ thick film, the lateral conductance of the film is well established as sensed by STM tunneling laterally through the film onto the sample holder at film edges.

STM images from the samples grown at three different times (or thicknesses) are shown in Fig. 1. Platinum film morphology appears to be a set of continuous mounds that become larger and more irregular as the film thickness increases. The observed morphology is very similar to the computer simulations by Amar and Family [23] for a growth model considering full surface diffusion, but is dissimilar to the morphology of large-scale uniformly sized pyramids as observed due to the Schwoebel barrier effect [19-22]. Usually, the Schwoebel barrier exists at the step edge of well-defined terraces as in an epitaxial growth starting with the layer growth mode. In a polycrystal growth starting with the randomly orientated grain growth mode and with grain sizes much smaller than film thickness, the Schwoebel barrier will be improbable.

In order to gain insight into the dynamic scaling behavior and detailed growth processes, we determined the scaling exponents and rms local slope. These quantities can be
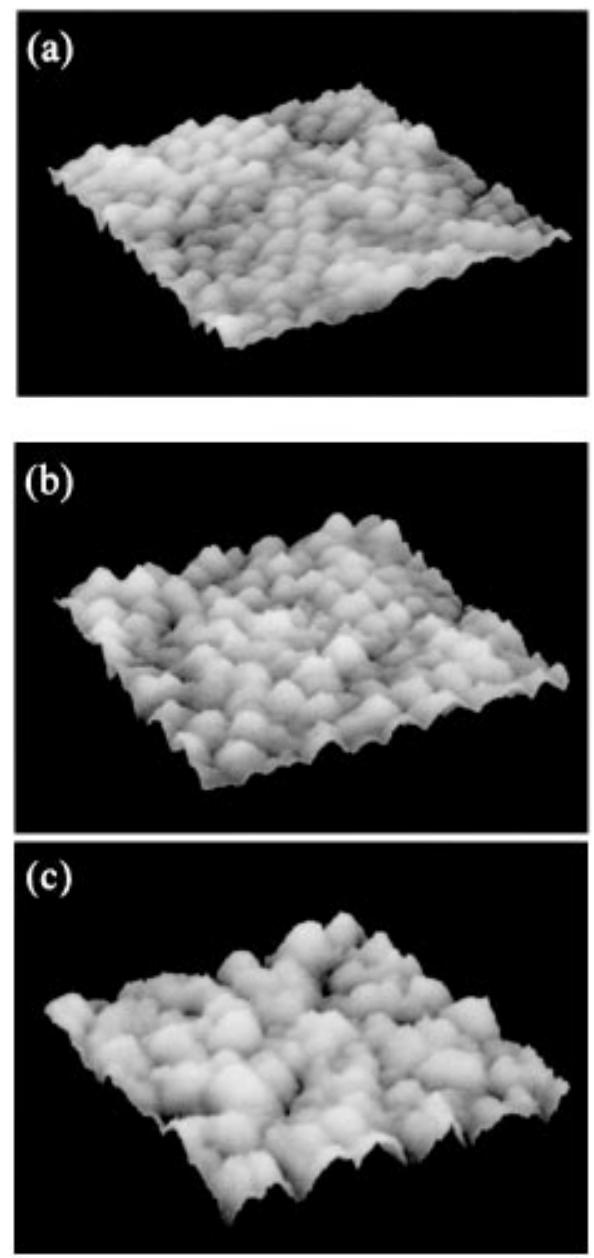

FIG. 1. Typical STM images for Pt films, grown at $t=25$, 58, and 108 min [from (a) to (c)], that show an evolution of the surface morphology as the film thickness increases. All image sizes are $100 \times 100 \mathrm{~nm}^{2}$, and actual vertical-axis scales are (a) 0-2 nm, (b) $0-3 \mathrm{~nm}$, and (c) $0-4 \mathrm{~nm}$. In order to enhance viewing, the vertical variations have been magnified by a factor of 4 .

obtained by calculating the height-height correlation function $G(\mathbf{r}, t)$, which is defined as the mean square of height difference between two surface positions separated by a lateral distance $\mathbf{r}$. If scaling exists, it is of the form $[1,2]$,

$$
\begin{aligned}
G(\mathbf{r}, t) & \equiv\left\langle[h(\mathbf{r}, t)-h(0, t)]^{2}\right\rangle \\
& =2 w(t)^{2} g(r / \xi(t))= \begin{cases}\rho(t)^{2} r^{2 \alpha} & \text { for } r \ll \xi(t), \\
2 w(t)^{2} & \text { for } r \gg \xi(t),\end{cases}
\end{aligned}
$$

with the rms roughness (or interface width) given by

$$
w(t)=\left\langle[h(\mathbf{r}, t)-\langle h\rangle]^{2}\right\rangle^{1 / 2} \propto t^{\beta},
$$

where $\langle\cdots\rangle$ is the spatial average over the sample surface, $\xi(t)$ is the correlation length which scales as $t^{\beta / \alpha}$, and $g(x)$ is a scaling function with the asymptotic behavior of $g(x) \propto \rho(t)^{2} x^{2 \alpha}$ for $x \ll 1$ and $g(x)=1$ for $x \gg 1$. If the rms local slope $\rho(t)$ is time invariant, the growing surface will exhibit a normal scaling behavior such as that predicted for the nonlinear diffusion process [2]. Otherwise, 
it will exhibit an anomalous scaling behavior such as predicted in Eq. (2) for the linear diffusion process. Shown in Fig. 2 is a $\log -\log$ plot of $G(\mathbf{r}, t)$ vs lateral distance $r$ for the Pt films grown at five different times. Statistical averaging is performed over multiple images of the same size, taken from different areas on the surface of each sample and also using different tips. From the figure we can see that $G(\mathbf{r}, t)$ increases linearly at small $r$ and plateaus at large $r$, consistent with the asymptotic behavior predicted in Eq. (3). The lateral position corresponding to the plateau point is equal to $\xi$, which is a measure of the average mound size. Where our observations differ from others reported in the literature $[10-12,14]$ is that we have directly observed an up-shift of $G(\mathbf{r}, t)$ as the film thickness increases. According to Eq. (3) for the small $r$ limit, this up-shift indicates an increase in the local slope $\rho(t)$ with growth time $t$. The value of $G(\mathbf{r}, t)$ at $r=1 \mathrm{~nm}$ is proportional to $\rho(t)^{2}$, and the determined $\rho(t)$ is plotted in Fig. 3 as a function of $t$. The solid curve in the figure is a leastsquares fit of Eq. (2) to the data with $\tau=8.69 \mathrm{~min}$ and $C=0.027$. As can be seen, the data can be well described by Eq. (2) predicted from the linear growth equation. The obtained transition time, $\tau=8.69 \mathrm{~min}$, implies that our thinnest film at $t=25 \mathrm{~min}(\sim 15 \mathrm{~nm}$ thick) has already entered the scaling regime. Amar and Family [23] have numerically calculated $G(\mathbf{r}, t)$ for a full surface diffusiondriven growth model and found a similar up-shift of $G(\mathbf{r}, t)$ with increasing time. However, in their calculations, the average lateral size of mounds does not increase, yet their mound height increases linearly. In our case, we have observed that the lateral growth of mounds is slower than their vertical growth as indicated by the increase of the local slope $\rho(t)$.

Next, we determined the scaling exponents $\alpha$ and $\beta$ in order to isolate the exact growth process in our system. The exponent $\alpha$ is obtained by least-squares fit-

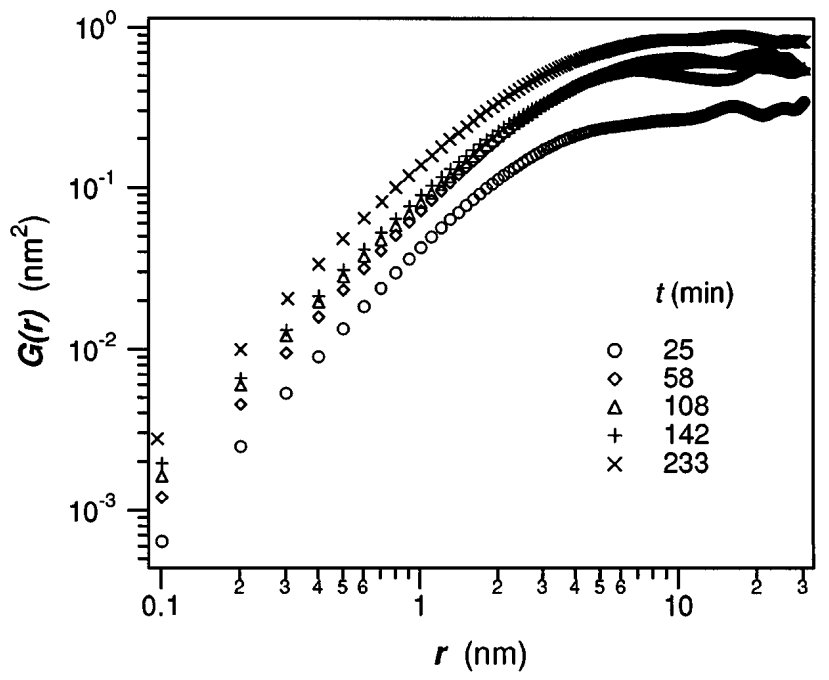

FIG. 2. A log-log plot of the height-height correlation functions $G(\mathbf{r}, t)$, calculated from STM images, as a function of lateral distance $r$ at five different growth times. ting to the linear slope, which gives $2 \alpha$, of $G(\mathbf{r}, t)$ at small $r(\leq 1 \mathrm{~nm})$ in Fig. 2. The obtained $\alpha$ 's for different thick films are plotted in Fig. 4(a) and are found to be independent of growth time within experimental uncertainty. This result indicates that the exponent $\alpha$ has reached its asymptotic limit. An average over these $\alpha$ 's gives a value of $0.9 \pm 0.02$ represented by the solid line in Fig. 4(a). The exponent $\beta$ is obtained by calculating the interface width $w(t)$, using the definition in Eq. (4), directly from STM images grown at different times, where the same statistical averaging as for $G(\mathbf{r}, t)$ has been employed. Figure 4(b) shows $w(t)$ vs $t$ in a $\log$-log scale and the slope gives $\beta=0.26 \pm 0.03$ as represented by the fitting line. All $w(t)$ 's in Fig. 4(b) have been corrected for an average substrate roughness of $w_{\text {glass }} \sim 0.17 \mathrm{~nm}$ according to $w_{\text {corrected }}=\sqrt{w^{2}-w_{\text {glass }}^{2}}$, where surface heights for both the substrates and Pt films follow Gaussian distributions. However, the value of $\beta$ changes $\sim 0.03$ before and after this correction. Obviously, the measured $\alpha$ and $\beta$ are in good agreement with the prediction $(\alpha=1$ and $\beta=0.25$ ) from the linear growth equation.

Based on the measured scaling exponents and the observed $\sqrt{\ln (t)}$ dependence of the local slope above, we can conclude that Pt sputter deposition on glass under our experimental conditions is governed by the linear diffusion process. This phenomenon arises from the fact that under the low growth temperature $(\sim 300 \mathrm{~K})$, deposited atoms can relax only to nearby kink sites and adhere there irreversibly. This local diffusion of atoms is thus unable to balance the fluctuations in incident flux, resulting in a growing local slope with time. The observed irregularly growing mounds could be initiated by random nuclei in early nucleation process. Although the exponents are also consistent with those predicted for the Schwoebel barrier model [17], the observed morphology differs from that in the Schwoebel barrier model in which the large-scale

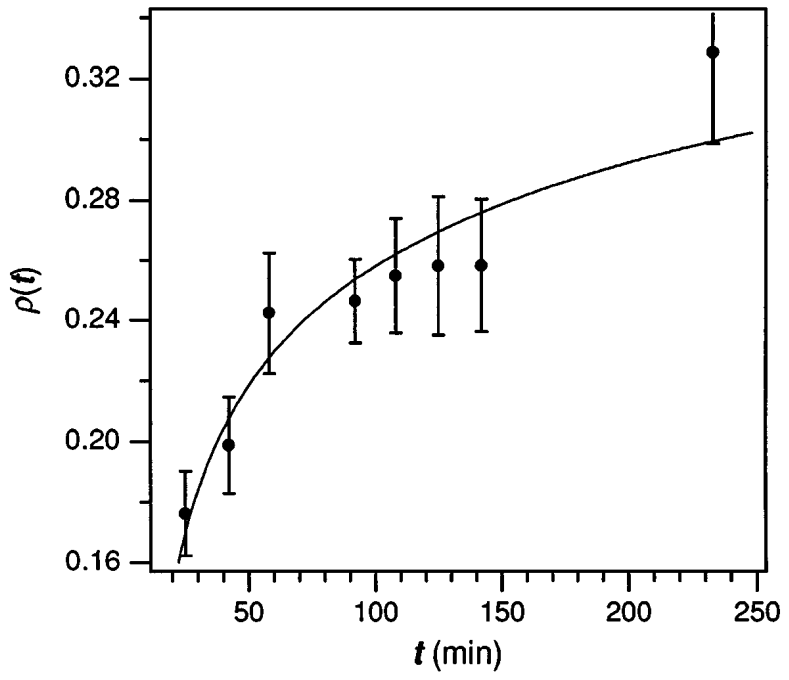

FIG. 3. A plot of the rms local slope $\rho(t)$ as a function of growth time $t$. The solid curve is a least-squares fit of Eq. (2) to the data. 


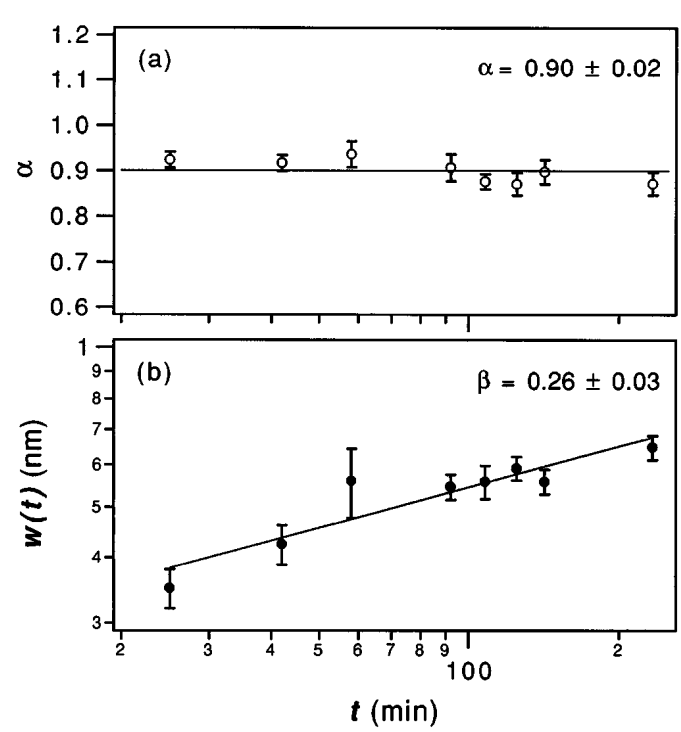

FIG. 4. The plots of the roughness exponent $\alpha$ and interface width $w(t)$ vs growth time $t$. (a) The line indicates an average value of $\sim 0.9 \pm 0.02$ over these data points. (b) Shows a power-law fit of $w(t)$ with $t$, and the slope gives $\beta$ as indicated by the fitting line.

pyramids of regular size will be formed. The slope of the pyramids will remain constant if the Schwoebel barrier height is considerable; otherwise, the slope will increase with time following a power law [24]. Finally, we would like to make a comparison, respectively, with two relevant STM experiments. One experiment concerns Au films sputter deposited on $\mathrm{Si}(111)$ with random incidence [12], a growth technique similar to ours except in that experiment the sample holder is fixed during deposition. In that study, the scaling exponents are measured to be $\alpha \sim 0.42$ and $\beta \sim 0.4$ at $300 \mathrm{~K}$. The inconsistency with our measured exponents could be due to a cooperation of the shadowing effect for the random incidence and surface diffusion in their case. The other STM experiment concerns Au films vapor deposited on glass at $298 \mathrm{~K}$ with oblique incidence [14], a system similar to ours. Because of the shadowing effect in the oblique incidence, the growth produced columns (height-enhanced mounds). The heightheight correlation function $G(\mathbf{r}, t)$ was found to exhibit two power-law regimes with $\alpha \sim 0.89$ for $r<\xi$ and $\alpha \sim 0.35$ for $r>\xi$, where $\xi$ is the average column size. The larger exponent at short length scales can be explained due to the local surface diffusion effect, yet the smaller exponent at large length scales is unclear. By contrast, our results are dissimilar to both the experiments above, indicating that either of the shadowing effects for the random and oblique incidence does not exist in our case, probably owing to the spinning and planetary rotation of our sample holder.

In summary, we have observed with STM a growth instability governed by the linear diffusion process occurring in Pt films sputter deposited on glass at room temperature. The evidence for this observation is given by the measured scaling exponents $(\alpha \sim 0.9$ and $\beta \sim 0.26)$ and $\sqrt{\ln (t)}$ dependence of the local slope. We exclude the Schwoebel barrier model due to different morphologies observed, and also the Schwoebel barrier effect is unlikely in polycrystalline systems with randomly orientated small grains.

We thank H.-N. Yang for invaluable discussions and the AFM imaging on our glass substrates. This research is supported by NSF Grant No. DMR-9311586.

[1] F. Family and T. Vicsek, Dynamics of Fractal Surfaces (World Scientific, Singapore, 1991).

[2] H.-N. Yang, T.-M. Lu, and G.-C. Wang, Diffraction from Rough Surfaces and Dynamic Growth Fronts (World Scientific, Singapore, 1993).

[3] A.-L. Barabási and H. Stanley, Fractal Concepts in Surface Growth (Cambridge University Press, Cambridge, England, 1995).

[4] Z.-W. Lai and S. Das Sarma, Phys. Rev. Lett. 66, 2348 (1991).

[5] D. E. Wolf and J. Villain, Europhys. Lett. 13, 389 (1990).

[6] S. Das Sarma and S. V. Ghaisas, Phys. Rev. Lett. 69, 3762 (1992); 71, 2510 (1993).

[7] J. G. Amar, P.-M. Lam, and F. Family, Phys. Rev. E 47, 3242 (1993).

[8] H.-N. Yang, G.-C. Wang, and T.-M. Lu, Phys. Rev. Lett. 73, 2348 (1994).

[9] Y.-L. He, H.-N. Yang, T.-M. Lu, and G.-C. Wang, Phys. Rev. Lett. 69, 3770 (1992).

[10] C. Thompson, G. Palasantzas, Y.P. Feng, S. K. Sinha, and J. Krim, Phys. Rev. B 49, 4902 (1994).

[11] G. Palasantzas and J. Krim, Phys. Rev. Lett. 73, 3564 (1994).

[12] H. You, R.P. Chiarello, H. K. Kim, and K. G. Vandervoort, Phys. Rev. Lett. 70, 2900 (1993).

[13] H. Zeng and G. Vidali, Phys. Rev. Lett. 74, 582 (1995).

[14] R. C. Salvarezza, L. Väzquez, P. Herrasti, P. Ocón, J. M. Vara, and A. J. Arvia, Europhys. Lett. 20, 727 (1992).

[15] W. M. Tong, R. S. Williams, A. Yanase, Y. Segawa, and M. S. Anderson, Phys. Rev. Lett. 72, 3374 (1994).

[16] P. P. Swaddling, D. F. McMorrow, R. A. Cowley, R. C. C. Ward, and M. R. Wells, Phys. Rev. Lett. 73, 2232 (1994).

[17] M. Siegert and M. Plischke, Phys. Rev. Lett. 73, 1517 (1994).

[18] J. A. Stroscio, D. T. Pierce, M. Stiles, A. Zangwill, and L. M. Sander, Phys. Rev. Lett. 75, 4246 (1995).

[19] M.D. Johnson, C. Orme, A. W. Hunt, D. Graff, J. Sudijono, L. M. Sander, and B. G. Orr, Phys. Rev. Lett. 72, 116 (1994).

[20] H.-J. Ernst, F. Fabre, R. Folkerts, and J. Lapujoulade, Phys. Rev. Lett. 72, 112 (1994).

[21] J. E. Van Nostrand, S. J. Chey, M.-A. Hasan, D. G. Cahill, and J.E. Greene, Phys. Rev. Lett. 74, 1127 (1995).

[22] K. Thürmer, R. Koch, M. Weber, and K. H. Rieder, Phys. Rev. Lett. 75, 1767 (1995).

[23] J. G. Amar and F. Family, in Mechanisms of Thin Film Evolution, edited by S. M. Yalisove, C. V. Thompson, and D. J. Eaglesham , MRS Symposia Proceedings No. 317 (Materials Research Society, Pittsburgh, 1994).

[24] P. Smilauer and D. D. Vvedensky, Phys. Rev. B 52, 14263 (1995). 